\title{
Projetando a Usabilidade e a Experiência do Usuário com a Técnica UXUG-AP: um Estudo Exploratório
}

\author{
Aline de Oliveira Sousa, Natasha Malveira Costa Valentim \\ Departamento de Informática - Universidade Federal do Paraná (UFPR) \\ Curitiba, PR - Brasil \\ ninimec@gmail.com, natasha@inf.ufpr.br
}

\begin{abstract}
Be different and distinguish itself among the technologies is intrinsic to the current agile software development. However, we still found usability and user experience (UX) defects generated by the process, making of the quality and user satisfaction unsatisfactory. Thu, this article presents a technique which support the usability and UX design in agile projects, calling User Experience and Usability Guidelines for Agile Project (UXUG-AP). Besides, this article presents a qualitative analysis and user perception analysis obtained through an exploratory study about the UXUG-AP technique. The results show that the technique is considered easy to use and useful.
\end{abstract}

Resumo. Ser diferente e se destacar em meio as opções tecnológicas é intrínseco ao desenvolvimento de software ágil atual. No entanto, ainda são encontrados defeitos de usabilidade e experiência do usuário (UX) gerado pelo processo, fazendo com que a qualidade e satisfação do usuário continuem insatisfatórios. Portanto, este artigo apresenta uma técnica que visa auxiliar no projeto da usabilidade e UX em projetos ágeis, chamada User Experience and Usability Guidelines for Agile Project (UXUG-AP). Além disso, este artigo apresenta uma análise qualitativa e uma análise da percepção do usuário obtidas através de um estudo exploratório sobre a técnica UXUG-AP. Resultados mostram que a técnica é considerada fácil de usar e útil.

\section{Introdução}

A todo momento, o processo de desenvolvimento de software evolui, a fim de atender de forma mais efetiva as demandas do mercado. Nos últimos anos, surgiram as metodologias ágeis [Beck et al. 2001], o que revolucionou a forma de desenvolvimento e promoveu entregas mais rápidas, bem como uma maior proximidade com o cliente final.

Além disso, as equipes de software buscam entender melhor a necessidade do usuário com o intuito de entregar um produto que proporcione maior satisfação no uso, além de ser um diferencial, dentre as soluções disponíveis. Por isso, inúmeros pesquisadores e profissionais vêm impulsionando a integração da usabilidade e experiência do usuário (User Experience - UX) no processo de desenvolvimento ágil.

A integração entre os conceitos de usabilidade e UX a metodologias ágeis ainda é imatura. Muitas são as propostas disponíveis na literatura. Algumas promovem a integração apenas entre metodologias ágeis e a usabilidade, outras apenas entre metodologias ágeis e UX. Elas ainda sugerem frameworks, processos, abordagens, dentre outras soluções. Muitas das propostas introduzem a usabilidade e UX ao final do processo de desenvolvimento, com avaliações e testes aplicados com os usuários finais usando o software já desenvolvido. Dessa forma, muitos defeitos são encontrados tardiamente na 
linha de tempo do processo de desenvolvimento, o que resulta em retrabalho e consequentemente aumento de custo e tempo. Para tanto, surge o questionamento: Como podemos projetar requisitos de usabilidade e UX ao mesmo tempo em projetos ágeis, evitando seus respectivos defeitos?

Portanto, neste artigo é apresentada a User Experience and Usability Guidelines for Agile Project (UXUG-AP), uma técnica que visa dar suporte ao projeto da usabilidade e UX em projetos ágeis no intuito de evitar possíveis defeitos de usabilidade e UX em fases posteriores do processo de desenvolvimento. Além disso, este artigo apresenta um estudo exploratório realizado com o intuito de avaliar o uso da técnica, bem como seu formato de apresentação e conteúdo.

O restante desse artigo está organizado em 5 seções. A Seção 2 aborda sobre os trabalhos relacionados e a Seção 3 apresenta a técnica UXUG-AP proposta. A Seção 4 aborda sobre o planejamento e execução do estudo exploratório realizado, assim como os resultados da análise da percepção dos participantes e análise qualitativa do estudo. Finalmente, a Seção 5 apresenta as conclusões, bem como os trabalhos futuros.

\section{Trabalhos Relacionados}

Memmel et al. (2007) apresentam um ciclo de vida Extreme Programming (XP) estendido com a Engenharia de Usabilidade. Eles utilizam a prototipação como parte do processo para discussão com os stakeholders bem como repositório dos requisitos. No entanto, também apresenta algumas lacunas como o uso de uma metodologia específica o que limita a utilização em outras metodologias. Além disso, usam apenas o conceito de usabilidade para a integração a metodologias ágeis.

Newhook et al. (2015) apresentam três estudos de caso, realizados dentro de um contexto agile, onde abordam o uso de design iterativo e reuso de artefatos de design em projetos da IBM que utilizam o modelo IBM Design Thinking. Todas as abordagens apresentadas foram especificamente desenvolvidas para produtos de software mobile. Os estudos concluíram que as expectativas do usuário têm criado uma barra na qual se constitui na experiência do usuário aceitável para empresas de aplicativos mobile. Também se concluiu que existe um novo foco em facilidade no uso e design visual que as empresas precisam atender ao mesmo tempo que atendem ao processo do negócio. No entanto, os estudos de caso focam apenas na plataforma mobile além de serem aplicados apenas no modelo de trabalho da IBM, não apresentando a liberdade na escolha da metodologia ágil a ser adotada ou na plataforma a ser aplicada.

Hussain et al. (2012) apresentam um processo que integra conceitos de Interação Humano Computador em um projeto que utilizava a metodologia XP. A abordagem incluiu múltiplos instrumentos como Estudos dos usuários, Testes de usabilidade, Avaliações de especialistas em UX e Testes de unidade estendido. Embora este estudo traga os conceitos tanto da usabilidade, quanto da UX, ele aplica estes conceitos no final do processo de desenvolvimentos ágil, o que torna qualquer alteração ou correção de defeitos mais cara, além do retrabalho gerado e do aumento de tempo até a entrega do produto ao usuário.

Diante das lacunas identificadas e citadas nos trabalhos relacionados acima, propôs-se a UXUG-AP, que será detalhada na seção a seguir. Inicialmente, no que diz respeito a metodologia ágil adotada, a UXUG-AP pode ser aplicável em qualquer metodologia ágil. Outro diferencial apresentado pela UXUG-AP é a simplicidade que ela 
demonstra ao utilizar a técnica de prototipação, quando comparada às propostas citadas anteriormente que necessitam de vários instrumentos e técnicas para serem utilizadas. Um adicional é o fato de trabalhar os conceitos de usabilidade e UX em conjunto e no início do processo de desenvolvimento, durante o design do produto.

\section{User Experience and Usability Guidelines for Agile Project}

A User Experience and Usability Guidelines for Agile Project (UXUG-AP) é uma técnica que possui a prototipação como base e que dá suporte às metodologias ágeis em relação ao projeto da usabilidade e UX. A UXUG-AP mantém características intrínsecas da metodologia ágil, como: (a) baixo custo, por não precisar de suporte ferramental ou artefatos que demandem materiais caros; (b) flexibilidade, por poder ser introduzida independente da metodologia ágil adotada; e (c) facilidade, por utilizar a técnica de prototipação como base, técnica bem difundida na indústria (Wale-Kolade, 2015; Shön et al. 2017; Kuusinen \& Mikkonen, 2014; Maguire, 2013; Isomursu et al. 2012; Karamanis et al. 2018). Para tanto, a técnica proposta pretende auxiliar os times ágeis a pensar e projetar seus softwares, visando aos requisitos de usabilidade e UX, com o objetivo de evitar possíveis defeitos de usabilidade e UX que normalmente são encontrados no final do processo de desenvolvimento.

A UXUG-AP [Sousa e Valentim, 2018] possui um conjunto de diretrizes. Ela é organizada em 11 categorias, onde cada categoria contém suas respectivas subcategorias e dentro destas subcategorias são apresentadas diretrizes e exemplos (Tabela 1).

Tabela 1. Categorias e Subcategorias da UXUG-AP

\begin{tabular}{|c|c|}
\hline Categorias & Subcategorias \\
\hline \multirow{3}{*}{ Requisitos } & Troca de Informação -Time/Cliente \\
\hline & Entrevistas e Workshops \\
\hline & Requisitos Chave \\
\hline \multirow{3}{*}{$\begin{array}{l}\text { Entendimento das Necessidades do } \\
\text { Usuário }\end{array}$} & Iniciantes e Especialistas \\
\hline & Crianças Jovens, Adultos e Idosos \\
\hline & Leigos, Acadêmicos e Profissionais \\
\hline \multirow{2}{*}{ Acessibilidade } & Deficientes Visual I \\
\hline & Deficientes Visual II \\
\hline Facilidade no Uso & Localização \\
\hline \multirow{4}{*}{ Feedback Informativo } & Mensagens de Confirmação e Alerta \\
\hline & Mensagens de Erro \\
\hline & Componentes de Carregamento \\
\hline & Títulos e Links \\
\hline \multirow{3}{*}{ Prevenção de Erros } & Obrigatoriedade dos Campos \\
\hline & Limitando Campos \\
\hline & Apresentação Autoexplicativa \\
\hline \multirow{2}{*}{ Agrupamento de Informação } & Independência da Informações \\
\hline & Modularização de Informação \\
\hline \multirow{2}{*}{ Sequência de Ações } & Organização das Ações Sequenciais \\
\hline & Comportamento das Ações Sequenciais \\
\hline Sentimento de Pertencimento & Conexão Emocional \\
\hline \multirow{2}{*}{ Grau de Importância } & Disposição das Informações \\
\hline & Termos Relevantes \\
\hline \multirow{2}{*}{ Privacidade } & Controle de Informação \\
\hline & Senhas \\
\hline
\end{tabular}


Através desta estrutura, os profissionais são capazes de consultar livremente o material a fim de adquirir ideias, utilizar os exemplos e principalmente despertar o pensamento crítico a respeito da usabilidade e UX do software a ser construído. O material também pode ser usado conforme a necessidade do projeto, não havendo a necessidade de consultar todas as categorias para uma mesma funcionalidade a ser desenvolvida.

Além dos trabalhos relacionados, utilizados para motivar a criação da técnica, outros trabalhos foram utilizados como base para criação da UXUG-AP, dando o suporte necessário e fornecendo todo o embasamento teórico para a mesma, como ISO/IEC 25010 (2011); Carvajal et al. (2013); Scheneiderman et al. (2016); Usabuility Guidelines (2019); Windows User Experience Interaction Guideline (2019); Tognazzini (2014) e Harley (2018). Conforme pode ser visto nas figura abaixo, a técnica UXUG-AP é apresentada através de um PDF clicável formada por links, onde o profissional pode selecionar a categoria desejada (Figura 1) e, ao ser direcionado para as respectivas subcategorias (Figura 2), ele pode selecionar a subcategoria que melhor lhe aprouver e, ao finalmente ser direcionado para a diretriz da subcategoria correspondente (Figura 3), ele pode lê-la e validar se a diretriz pode contribuir para a construção do seu software.

Por exemplo, a partir da leitura de uma estória do usuário, pode-se começar a utilização da técnica através do acesso à categoria "Requisitos", de forma que, ao ler suas respectivas subcategorias, a técnica auxilia na compreensão da importância dos requisitos, além de instigar o pensamento do profissional em busca de respostas à perguntas como: Quem é o usuário que utilizará o produto final? Qual é a sua real necessidade? Qual a melhor forma de garantir sua satisfação no uso?

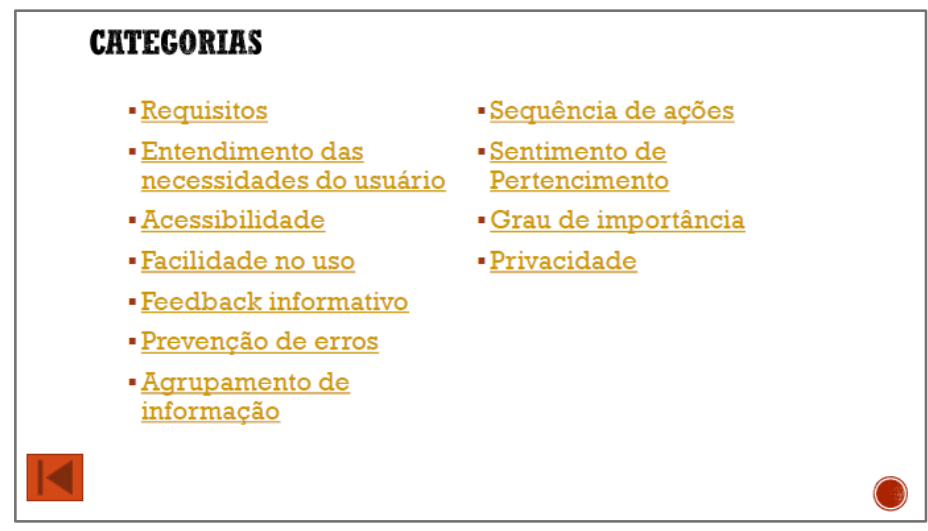

Figura 1. Categorias da UXUG-AP

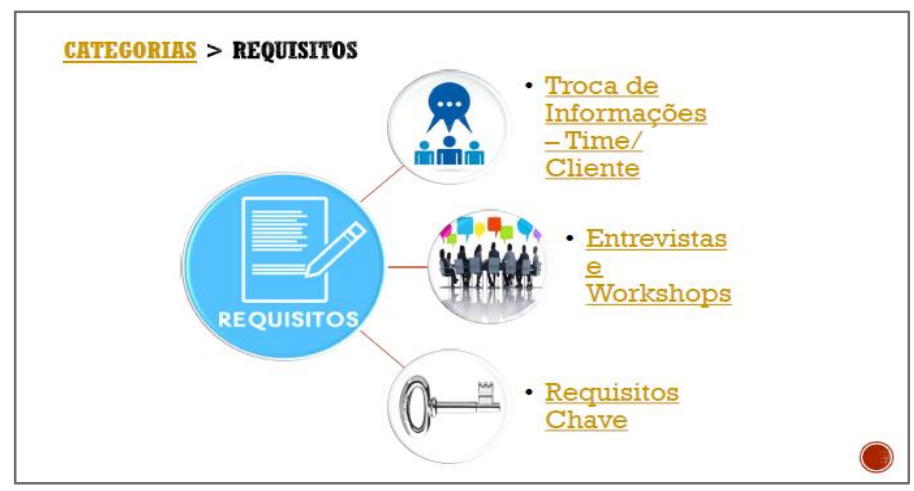

Figura 2. Subcategorias da UXUG-AP 


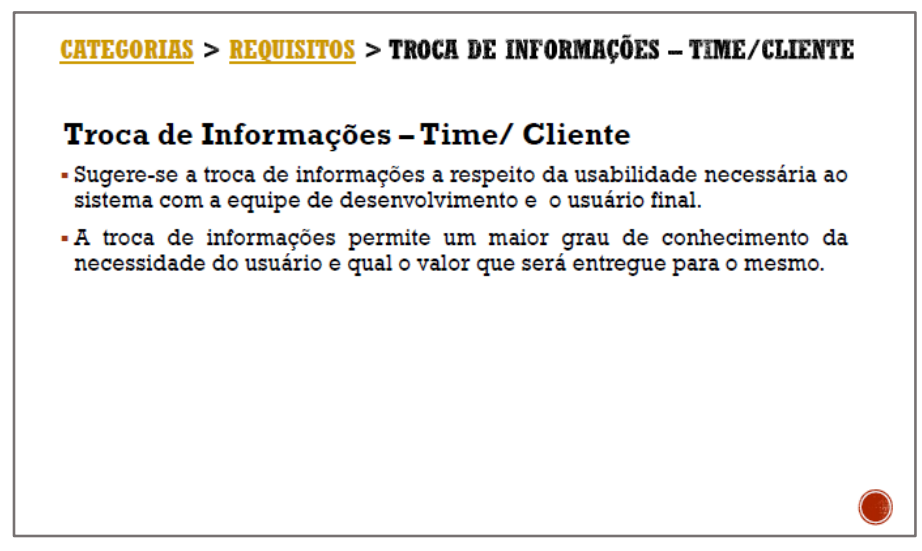

Figura 3. Diretrizes da UXUG-AP

Além disso, vale ressaltar que a técnica não apresenta necessidade de suporte ferramental, visto que a prototipação de baixa fidelidade pode ser feita em papel, por exemplo. $\mathrm{O}$ conhecimento específico para sua utilização também não é necessário, visto que no contexto ágil, a maioria dos times já estão acostumados com a prototipação.

\section{Estudo Exploratório}

\subsection{Planejamento e Execução do Estudo}

Foi realizado um estudo exploratório com estudantes de graduação da disciplina de Engenharia de Software de uma Universidade Pública, o qual teve como objetivo avaliar se a técnica UXUG-AP foi eficaz e eficiente ao dar suporte ao projeto da usabilidade e UX dentro de um ambiente ágil, além de avaliar sua facilidade de uso, utilidade percebida e intenção de uso futuro. O estudo contou com um total de 10 participantes que utilizaram a técnica UXUG-AP como apoio para a prototipação. Todos os participantes apresentavam conhecimento básico sobre metodologias ágeis, usabilidade e UX, pois estavam cursando a disciplina de Engenharia de Software. No entanto, estes participantes não possuem experiência profissional.

Os participantes receberam um Termo de Consentimento Livre e Esclarecido (TCLE) a fim de garantir o anonimato de seus dados e para obtermos a autorização da participação deles. Também foi entregue uma lista de instruções contendo os passos que seriam seguidos no decorrer da atividade. Uma estória do usuário foi entregue contendo os requisitos base para a construção dos protótipos. Após a execução da atividade, foi entregue um Questionário Pós-Uso, contendo as afirmações do Technology Acceptance Model (TAM) [Davis et al. 1989], no qual os participantes poderiam expor sua aceitação em relação à técnica considerando três indicadores: Facilidade no Uso, Utilidade Percebida e Intenção de Uso Futuro. Além disso, este Questionário Pós-Uso continha espaço para os participantes fornecerem comentários adicionais sobre a técnica.

\subsection{Análise da Percepção dos Participantes usando TAM}

As técnicas foram avaliadas pelos participantes em relação aos 3 indicadores do TAM, sendo eles Facilidade no Uso, Utilidade Percebida e Intenção de Uso Futuro. Entende-se Facilidade no Uso como a expressão das expectativas do usuário onde ele possa ter uma diminuição do esforço durante a execução de uma atividade. Já a Utilidade Percebida é entendida como o quanto uma pessoa acredita que seu desempenho pode ser melhorado 
a partir do uso de uma determinada tecnologia. Enquanto isso, a Intenção de Uso Futuro é consequência dos dois indicadores anteriores, de forma que quando uma tecnologia é percebida como fácil durante a utilização, além de útil, faz com que a tecnologia se torne mais interessante, aumentando a intenção de uso futuro da mesma.

As afirmações do indicador Facilidade de Uso são representadas pelos códigos: (FU1) Minha interação com a UXUG-AP foi clara e compreensível; (FU2) Interagir com a UXUG-AP não exige muito do meu esforço mental; (FU3) Considero a UXUG-AP fácil de usar; e (FU4) Considero fácil utilizar a UXUG-AP para fazer o que eu quero que ela faça, apoiar a construção de protótipos visando a usabilidade e experiencia do usuário da aplicação final. As afirmações do indicador Utilidade Percebida são representadas pelos códigos: (UP1) Usar a UXUG-AP melhorou o meu desempenho na construção de protótipos com boa usabilidade e experiência do usuário; (UP2) Usar a UXUG-AP permitiu aumentar a minha produtividade na construção de protótipos com boa usabilidade e UX; (UP3) Usar a UXUG-AP aumentou minha eficácia na construção de protótipos com boa usabilidade e UX; e (UP4) Considero a UXUG-AP útil para a construção de protótipos visando a utilidade e UX da aplicação final. As afirmações do indicador Intenção de Uso Futuro são representadas pelos códigos: (UF1) Supondo que eu tenha acesso a UXUG-AP, eu pretendo usá-la; e (UF2) Levando em conta que eu tenho acesso a UXUG-AP, eu prevejo que eu iria usá-la.

Nos resultados, os participantes serão apresentados a partir de códigos, de forma que "P" representa o indicativo de participante e será seguido por um número que representará o número do participante, por exemplo "P1". As escalas de cores utilizadas indicam o nível de concordância, como: Concordo totalmente (verde mais escuro), Concordo Amplamente (verde médio), Concordo Parcialmente (verde mais claro), Não concordo Nem discordo (Amarelo), Descordo parcialmente (amarelo escuro), Discordo amplamente (laranja) e Discordo totalmente (vermelho mais escuro). Na Gráfico 1 são apresentadas as percepções de cada participante em relação a cada afirmação de um respectivo indicador, conforme apresentado acima.

Gráfico 1. Percepção dos Participantes sobre a UXUG-AP

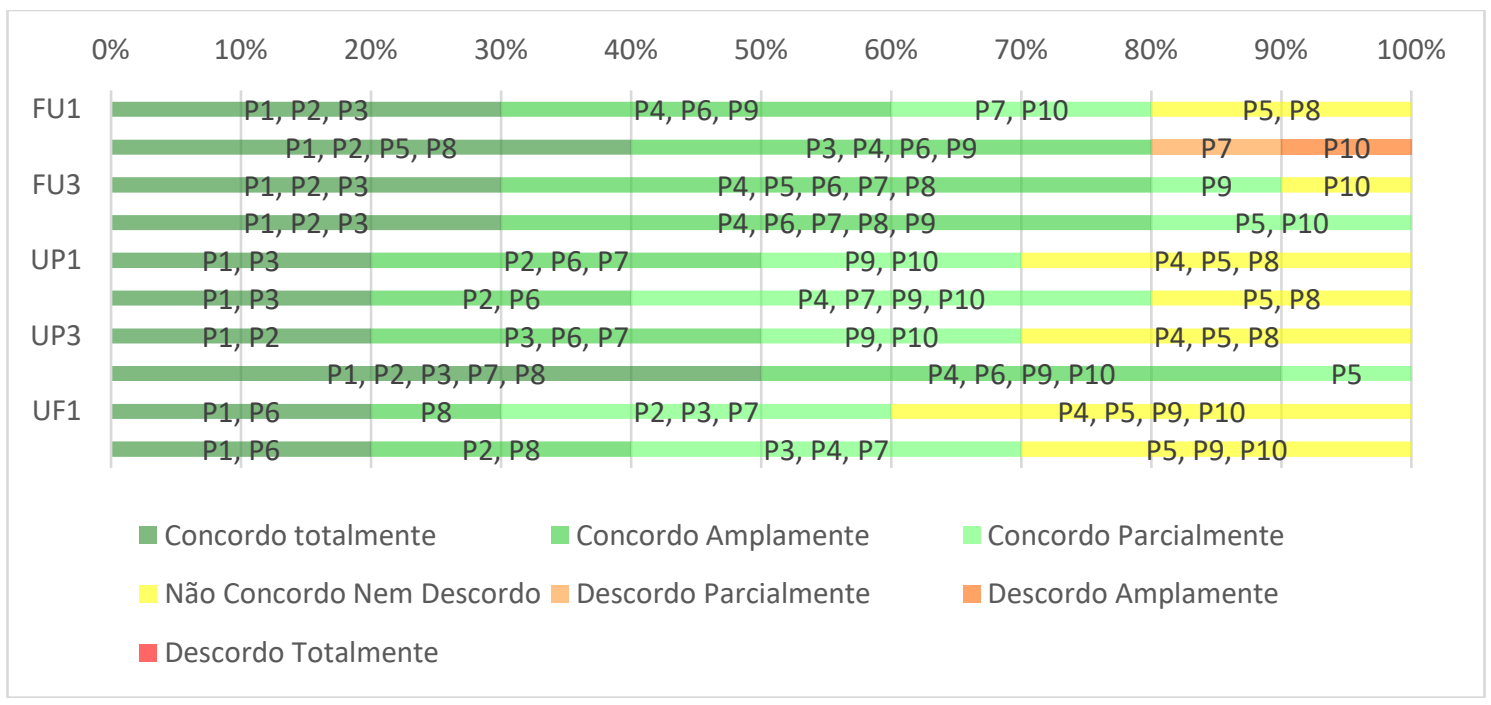

A partir dos dados colhidos através do Questionário Pós-Uso, no geral, observouse que a maioria dos participantes se apresentaram positivos ao utilizarem a técnica, pois concordaram totalmente ou amplamente com as afirmações referentes a Facilidade no 
uso, Utilidade e Intenção de Uso Futuro.

Porém, também é possível observar a discordância dos participantes P7 e P10 na a afirmação "(FU2) Interagir com a UXUG-AP não exige muito do meu esforço mental". Isso demonstra que existe a necessidade de deixar a técnica mais clara e didática, a fim de diminuir o esforço mental durante a utilização da técnica. Uma possível forma de preencher esta lacuna poderia ser a adição de mais exemplos, descritos em linguagem mais simples, ou até mesmo a adição de elementos visuais a fim de facilitar a compreensão e imaginação do usuário durante o uso da técnica.

Por outro lado, alguns participantes também apresentaram uma opinião imparcial, ou seja, não concordando nem discordando das afirmações apresentadas. Isso sugere que talvez a utilidade da técnica não tenha fícado tão explícita durante o uso da mesma. No entanto, isso também poderia ser explicado devido ao fato do estudo ter sido realizado com estudantes, os quais não apresentavam muito conhecimento prático de design de software e desenvolvimento ágil, gerando assim uma visão mais limitada do uso da técnica e de sua aplicabilidade em um contexto real.

\subsection{Análise Qualitativa}

Para a análise qualitativa foram utilizadas algumas das etapas do método Grounded Theory (GT), onde foram aplicadas três etapas, conforme sugerido por Corbin e Strauss (1990). A primeira etapa consiste na coleta e análise dos dados qualitativos. Posteriormente, na segunda etapa, os dados analisados são separados em códigos (unidade básica de análise que pode ser utilizada para indicar mais de um dado qualitativo, ou seja, o mesmo código pode ser utilizado para descrever os dizeres de mais de um participante, desde que estes estejam falando sobre o mesmo fenômeno). Posteriormente, na terceira etapa, os códigos que se relacionam ao mesmo fenômeno são agrupados e separados em categorias, e avalia-se os relacionamentos entre estes. Para a análise com GT, foram utilizados os comentários adicionais que os participantes fizeram no Questionário Pós-Uso.

Dessa forma, inicialmente separaram-se todos os dados qualitativos. Então, estes dados foram analisados para finalmente serem codificados, ou seja, foram separadas todas as frases relevantes e criados códigos que representassem estas frases. Em um segundo momento, os códigos foram analisados e agrupados em categorias, tomando como base o contexto e conteúdo abordados. Finalmente, foi realizada uma análise de cada categoria, com seus respectivos códigos, a fim de compreender qual a percepção dos estudantes ao utilizar a técnica e, através deste entendimento, definir quais os pontos de melhoria necessários a técnica a fim de evoluir a mesma.

Após as análises foram identificadas 6 categorias no total, sendo elas: Sugestões, Utilidade, Benefícios, Resultados, Características e Nível de dificuldade. A categoria Sugestões, apresentou duas características dominantes, sendo uma em relação ao exemplo de uso da UXUG-AP e outra em relação a adoção de um framework em conjunto com a técnica. O participante P10 sugeriu "Uma abordagem mais clara do funcionamento utilizando, por exemplo, um exemplo" ou "Uma explicação mais detalhada do processo de criação de um protótipo, com algum exemplo de uso, facilitaria o entendimento da técnica". Ou seja, um dos participantes sentiu a necessidade de existir um exemplo de uso a fim de clarificar os passos a serem seguidos durante o uso da UXUG-AP. Além disso, o participante P8 sugeriu “.... inclusão de frameworks como Bootstrap, Font Aewsome e 
Angular na UXUG-AP para auxiliar na prototipação". Isso indicou que o participante sentiu falta de sugestões mais visuais, como exemplos de botões ou campos.

Enquanto isso, a categoria Resultados indicou que a técnica foi satisfatória o suficiente fazendo com que alguns participantes vislumbrassem seu uso em algumas situações reais, como "A acessibilidade e o protótipo apresentado ao usuário final pode satisfazer melhor o cliente" dito pelo participante P2, ou ainda "Creio que seria usada no mercado" dito por P5.A categoria Características apresentou a satisfação dos participantes ao utilizarem a técnica, fato destacado por algumas características da mesma, como "É uma técnica muito ágil e concisa, onde ajuda e muito nos vários aspectos da criação dos protótipos" dita por P1, "Uma técnica simples e razoavelmente efetiva para prototipar um sistema com bom UX" dita por P9, "A técnica entrega o mesmo resultado das demais técnicas de prototipação, porém, por ser mais direta, torna a aplicação mais fácil, fazendo com que seu reuso seja inevitável" dito pelo participante P4 e "Cada categoria foi bem descrita" dita por P7.

Já a categoria Nível de dificuldade sugere a necessidade de tornar o método da UXUG-AP um pouco mais didático, principalmente quando o usuário não tem um conhecimento muito grande em relação a prototipação de software como era o caso dos participantes que possuíam apenas o conhecimento passado em sala de aula, conforme podemos ver em "Como o meu entendimento quanto a forma de usá-lo não ficou claro, não posso concordar plenamente com as informações, mas com o entendimento da técnica consegui usá-la como guia" ditas pelo participante P10.

Em contrapartida, também foram destacados os Benefícios e Utilidade da técnica, como "A UXUG-AP foca em outros pontos 'chave' que fazem com que o protótipo fique muito melhor representado" e "Muitos dos pontos levantados como disposição dos itens, cor e tamanho dos textos, linguagem, entre outros, normalmente esquecidos em uma implementação" ditas pelo participante P1, "A técnica é muito útil para desenvolvimento de sistemas web e mobile, por ser especificamente prototipação" dita por P8 e "O foco que a técnica dá a diferentes grupos de pessoas é um ponto chave para a sua utilidade" dita pelo participante $\mathrm{P} 3$, respectivamente.

\section{Conclusões e Trabalhos Futuros}

Neste artigo foi apresentada a técnica UXUG-AP que pretende dar suporte ao projeto da usabilidade e UX em projetos ágeis durante a prototipação do software. Além disso, também foram apresentados os resultados do estudo exploratório realizado com 10 alunos da graduação na disciplina de Engenharia de Software.

A análise da percepção dos participantes utilizando o TAM [Davis et al.]89] mostrou que, de forma geral, os participantes conseguiram utilizar facilmente a técnica, sem encontrar muitas dificuldades durante o manuseio desta. Já em relação a utilidade da UXUG-AP, boa parte dos participantes acreditam que a técnica seja útil ao que se propõe, ou seja, a dar suporte ao projeto da usabilidade UX em projetos ágeis. Em contrapartida, boa parte dos participantes destacaram a não intensão de uso futuro. No entanto, um dos possíveis motivos para este resultado poderia ser explicado pelo fato de se tratarem de estudantes que ainda não possuem uma atuação efetiva na indústria de software.

Enquanto isso, através da análise qualitativa, pode-se perceber que os participantes conseguiram enxergar algumas das características pretendidas na proposta da técnica, como agilidade e simplicidade. Além disso, os participantes apresentaram 
sugestões pertinentes que podem melhorar e facilitar o entendimento e utilização da técnica. De forma geral, pode-se concluir que apesar dos benefícios e utilidades encontrados, a UXUG-AP ainda necessita evoluir no quesito facilidade no uso, tornar-se mais didática, além da necessidade de exemplos, ou ainda a inclusão de frameworks.

Sendo assim, a UXUG-AP pode ser uma ferramenta de apoio no projeto da usabilidade e UX dentro de um contexto ágil. No entanto, ainda é necessário evoluir a técnica a fim de maximizar seu potencial objetivo que é evitar possíveis defeitos de Usabilidade e UX. Com isso, como trabalho futuro, espera-se evoluir a técnica com base nos dados alcançados, bem como aplicar um estudo de observação, com profissionais que atuem na área, a fim de avaliar a técnica evoluída.

\section{Referências}

Beck, K., Beedle, M., Van Bennekum, A., Cockburn, A., Cunningham, W., Fowler, M., Grenning, J., Highsmith, J., Hunt, A., Jeffries, R., Kern, J., Marick, B., Martin, R. C., Mellor, S., Schwaber, K., Sutherland, J. e Thomas, D. (2001) "Manifesto para desenvolvimento de software ágil”. Disponível em $<\mathrm{http}$ ://agilemanifesto.org/>

Carvajal, L., Moreno, A. N., Sanchez-Segura, M. I. e Seffah, A. (2013) "Usability through Software Design", IEEE Transactions Software Engineering, vol. 39, n11, pp.15821596.

Corbin, J. e Strauss, A. (1990) "Grounded Theory Research: Procedures", Canons, and Evaluative Criteria. Qualitative Sociology, vol.13, n. 1, pp 3-21.

Davis, F. D., Bagozzi, R. P. e Warshaw, P. R. (1989) "User acceptance of computer technology: A comparison of two theoretical models", Management Science,35(8): 982-1003.

Harley, A. (2018) "UX Guidelines for Recommended Content", Disponível em: $<$ https://www.nngroup.com/articles/recommendation-guidelines/>.

Hussain, Z., Lechner, M., Milchrahm, H., Shahzad, S., Slany, W., Umgeher, M., Vlk, T., Köfel, C., Tscheligi, M. e Wolkerstorfer, P. (2012) "Practical Usability in XP Software Development Process", The Fifth International Conference on Advancesin ComputerHuman Interactions. pp. 208-217. Valencia, Espanha.

ISO/IEC (2011), "ISO/IEC 25010: Systems and software engineering - SquaRE Software Product Quality Requirements and Evaluation: System and Software Quality Models"

Isomursu, M., Sirotkin, A., Voltti, P. e Halonen, M. (2012) “User Experience Design Goes Agile in Lean Transformation - A Case Study", 2012 Agile Conference, pp. 1-10. Dallas, Texas.

Karamanis, N., Pignatelli, M., Silva-Carvalho, D., Rowland, F., Cham, J. A. e Dunhan, I. (2018), "Designing and Intuitive web application for drug Discovery scientist", Drug Discovery To-day, vol3, pp. 1169-1174.

Kuusinen, K. e Mikkonen, T. (2014) “On Designing UX for Mobile Enterprise App", 40th Euro micro Conference on Software Engineering and Advanced Applications. Verona, Italia.

Maguire, M. (2013) "Using human factors standards to support user experience and agile 
design", Stephanidis, C. and Antona, M. (eds.). $7^{\text {a }}$ International UAHCI Conference, HCI International, pp. 185-194. Las Vegas, USA.

Memmel, T., Reiterer, H. e Holzinger. A. (2007) "Agile Methods and Visual Specification in Soft-ware Development: A Chance to Ensure Universal Access", 2007 HCI Conference, pp.453-462.

Newhook, R., Jaramillo, D., Temple, J. G. e Duke, K. J. (2015) "Evolutions of the mobile enterprise app: A design perspective", International Conference on Applied Human Factors and Ergo-nomics and Affiliated Conferences. Procedia Manufacturing 3, pp. 2026-203.

Scheneiderman, B., Plaisant, C., Cohen, M., Jacobs, S. e Elmgvist, N (2016) "Designing the User Interface: Strategies for Effective Human-Computer Interaction". 6 a Edição, Pearson, http://www.cs.umd.edu/hcil/DTUI6.

Schön, E. M., Thomaschewski, J. e Escalona, M.J. (2017) "Agile Requirements Engineering: A systemic literature review”, Computer Standards \& Interfaces, vol. 49, pp. 79-91.

Sousa, A. O., Valentim, N. M. C. Técnica User Experience and Usability Guidelines for Agile Project (2018), https://drive.google.com/file/d/16EOb9EqzvCkCsqDviuot5_YrJXAIHZMZ/view?us $\mathrm{p}=$ sharing

Tognazzini, B. (2014) "First Principles of Interaction Design (Revised \&Expanded)", https://www.nngroup.com/articles/first-principles-interaction-design/.

Usability Guidelines. (2019) U.S. Departament of Health \& Human Services, Disponível em $<$ https://webstandards.hhs.gov/guidelines/>

Wale-Kolade, A. Y. (2015) "Integrating usability work into a large inter-organizational agile development project: Tactics developed by usability designers", The journal of Systems and Soft-ware, pp. 54-66.

Windows User Experience Interaction Guideline (2019), Microsoft Corporation. Disponível em: $\quad<$ https://docs.microsoft.com/enus/windows/desktop/uxguide/guidelines> 